\title{
Comparison of difference in muscle activity ratio, ground reaction force and knee valgus angle during single leg squat and landing according to dynamic taping
}

\author{
Tae-Won Ha ${ }^{a}$, Sam-Ho Park ${ }^{a(1)}$, Myung-Mo Lee ${ }^{b(1)}$ \\ ${ }^{a}$ Department of Physical Therapy, Graduate School, Daejeon University, Daejeon, Republic of Korea \\ ${ }^{\mathrm{b}}$ Department of Physical Therapy, Daejeon University, Daejeon, Republic of Korea
}

Objective: This study examined the effects of dynamic tape applied to the patellofemoral joint on the knee valgus angle, muscle activity, and ground reaction force during a single leg squat (SLS) and single leg landing (SLL).

Design: Cross-sectional study.

Methods: Twenty-four subjects (11 male, 13 female) who met the inclusion criteria were screened by the knee palpation and patella compression tests. First, the knee valgus angle and muscle activity during SLS were measured. Second, the knee valgus angle and ground reaction force during SLL were measured. For the intervention, a patella joint loop using dynamic tape was used. The knee valgus angle, muscle activities in SLS and SLL after the intervention, and the ground reaction force were measured in the same way. A paired t-test was used to examine the difference between before and after the intervention.

Results: The knee valgus angle showed a statistically significant improvement after dynamic taping application in SLS and SLL $(p<0.05)$. The differences in muscle activity of the VL/VMO and ground reaction forces were not statistically significant after dynamic taping application in SLS and SLL.

Conclusions: This study showed that dynamic taping applied around the patellofemoral joint was effective in improving the knee valgus angle in SLS and SLL and had a reduced risk of secondary injury during sports activity.

Key Words: Athletic tape, Electromyography, Genu valgum, Patellofemoral pain syndrome, Vertical dimension

\section{Introduction}

Patellofemoral pain syndrome is defined as pain in the anterior aspect of the knee, which is caused by problems with the patellofemoral joint itself or with the adjacent soft tissues [1]. The main symptom of patellofemoral pain syndrome is increased pain in postures where the load on the patellofemoral joint is increased, such as when ascending or descending stairs, walking on a ramp, squatting, or sitting for a long time [2]. When performing a single leg squat (SLS) and landing, the patella movement and tilting is biased to the lateral side, resulting in an increase in abnormal load and valgus angle [3].
Muscular imbalance in the quadriceps muscle causes patella dislocation and abnormal biomechanics [4]. In particular, the vastus medialis obliques (VMO), which is weaker than the vastus lateralis (VL), causes excessive lateral gliding, lateral tilt, and lateral rotation of the patella, resulting in increased pressure and dysfunction on the facies patellaris femoris [5]. Accordingly, strengthening the VMO is considered a key factor in a rehabilitation program to prevent abnormal tracking of the patella [6].

Squats are a typical closed kinetic chain exercises, and it is suggested to be an exercise method to strengthen the VMO of patients with patellofemoral pain syndrome [1]. Among them, SLS is used as an effective exercise for knee

Received: 30 November, 2020 Revised: 23 December, 2020 Accepted: 24 December, 2020

Corresponding author: Myung-Mo Lee (ORCID https://orcid.org/0000-0002-2192-1701)

Department of Physical Therapy, Daejeon University, 62 Daehak-ro, Dong-gu, Daejeon 34520, Republic of Korea

Tel: 82-42-280-4295 Fax: 82-42-280-2295 E-mail: mmlee@dju.kr

(c) This is an Open-Access article distributed under the terms of the Creative Commons Attribution Non-Commercial License (http://creativecommons.org/licenses/ by-nc/4.0) which permits unrestricted non-commercial use, distribution, and reproduction in any medium, provided the original work is properly cited.

Copyright @ 2020 Korean Academy of Physical Therapy Rehabilitation Science 
rehabilitation due to its ability to produce higher muscle activity than squat exercises [7]. Therefore, various physical exercises are required to rehabilitate the knee and resolve the imbalance of the quadriceps muscles. Single leg landing (SLL) is a frequently performed movement during such sports activities, but incorrect SLL increases knee valgus and causes damage around the patellofemoral joint [8].

To solve these problems, clinical practice involves applying a taping method to prevent and assist the abnormal tracking of the patella [9]. Taping methods can be classified into 2 types depending on the kind of tape: rigid and elastic tape [10]. Dynamic tape, a type of elastic tape introduced by the Australian physiotherapist Ryan Kendrick, has elasticity and can be stretched by more than $200 \%$, as well as resistance and rebound stretching abilities in both the vertical and transverse directions [11]. Therefore, it is considered that dynamic taping can effectively prevent injury and maintain a stable position from excessive load and impact on the patellofemoral joint during sports activities. This study measured and compared the VMO/VL muscle activity ratio, knee valgus angle, and vertical ground force according to the application of dynamic taping during SLS and SLL in adults with patellofemoral pain, as long as excessive load and impact on the patellofemoral joint occurred.

\section{Methods}

\section{Participants}

The participants in this study were young adults with patellofemoral pain attending Daejeon University located in Daejeon, Korea. The inclusion criteria of the study participants were as follows: those aged 18 to 40 years old, had experienced 3 or more incidences of patellofemoral pain within the last 6 months on the Visual Analog Scale (VAS), had experienced pain during palpation or compression around the patella, and could maintain the posture for more than 3 seconds after SLL. Those who had 3 or more incidences of pain in the ankle within 6 months on the VAS, had surgery within 1 year, complained of fear of falling during SLS or SLL, and had allergic reactions to the adhesive component were excluded. For the recruitment of research participants, 120 people were notified through a Google questionnaire, and 62 people who met the inclusion criteria were recruited. Of the 62 recruited, 24 patients who had pain in the palpation and compression tests were selected.

\section{Procedures}

This study was a cross-sectional study design. The study was conducted with 24 participants who met the inclusion criteria. The subjects appointed the painful leg as the test leg; the dominant leg was appointed as the test leg if the pain was bilateral. Subjects practiced SLS and SLL postures 3 to 5 times with the examiner's assistance before the measurement. To compare the effects of the dependent variables before and after the intervention, 24 subjects were pre-tested and posttested by applying dynamic taping. This study was conducted after obtaining approval from the Institutional Bioethics

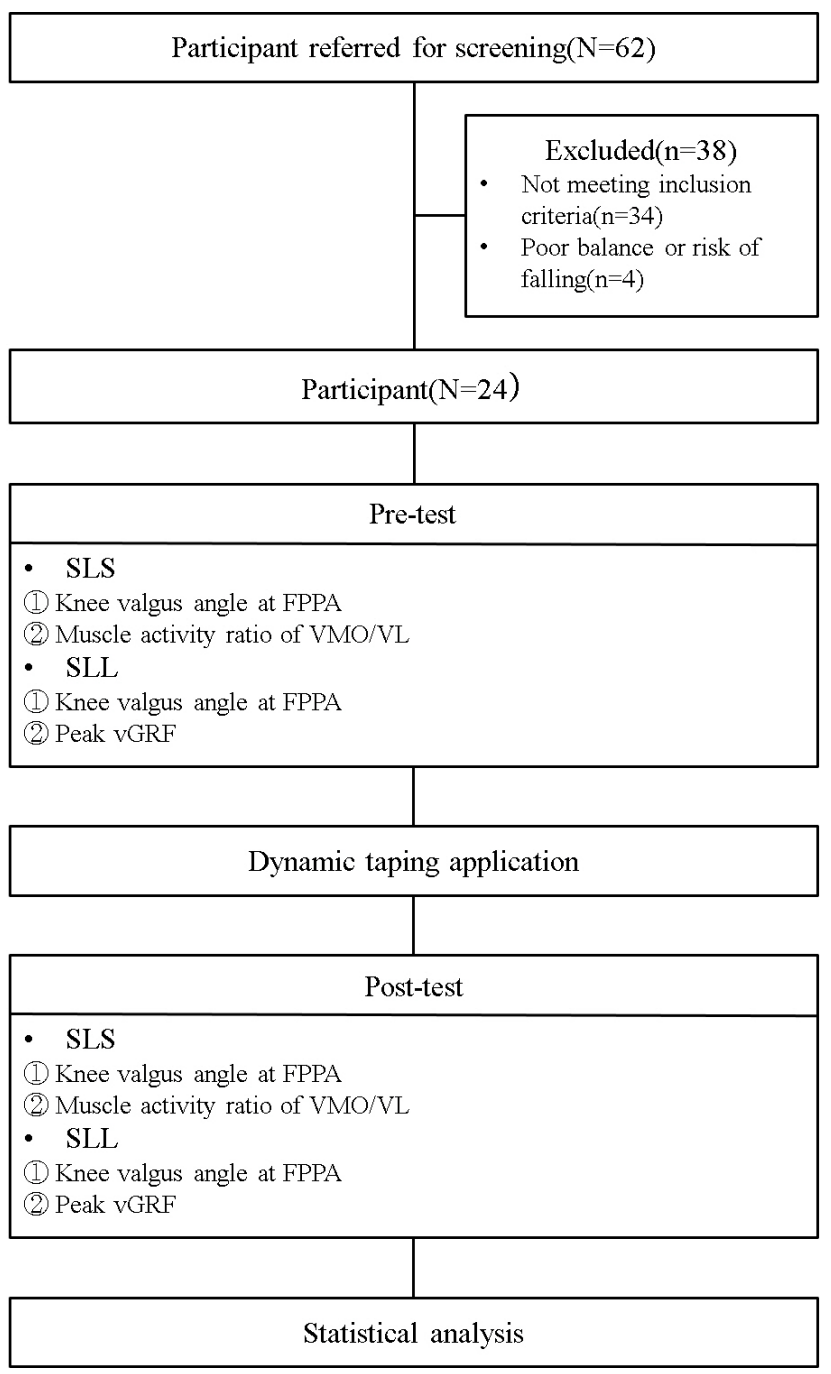

Figure 1. Flow chart. SLS: single leg squat, FPPA: frontal plane projection angle, VMO: vastus medialis obliques, VL: vastus lateralis, SLL: single leg landing, vGRF: vertical ground reaction force. 
Committee of Daejeon University. The research procedure was as follows (Figure 1).

\section{Dynamic taping}

In this study, a 'patella joint loop' technique was applied using Dynamic Tape Eco (Dynamic Tape, Port Vila, Vanuatu). The examiner prepared the tape that measured at a distance from the middle of the participant's VMO to the inferior angle of the patella. The middle was cut so that $2 / 3$ of the width of the tape could be used as the lateral parts and $1 / 3$ as the medial parts. The examiner began to apply the tape to the VMO at the middle of the hip and knee joints. The tape was attached in the downward and lateral directions. The angle was made so that the patella could receive force from the superior to the medial direction. The medial parts were attached along the direction of the VL and rotated around the knee bone. At this time, care was taken not to contact the patella directly. The lateral parts were pushed so that the patella could glide medially when attached by rotating around the patella (Figure 2). After applying dynamic taping, the sub-

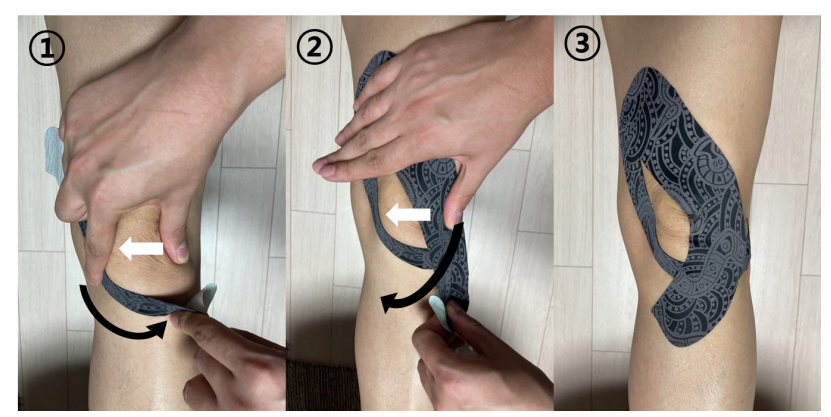

Figure 2. Dynamic taping application.
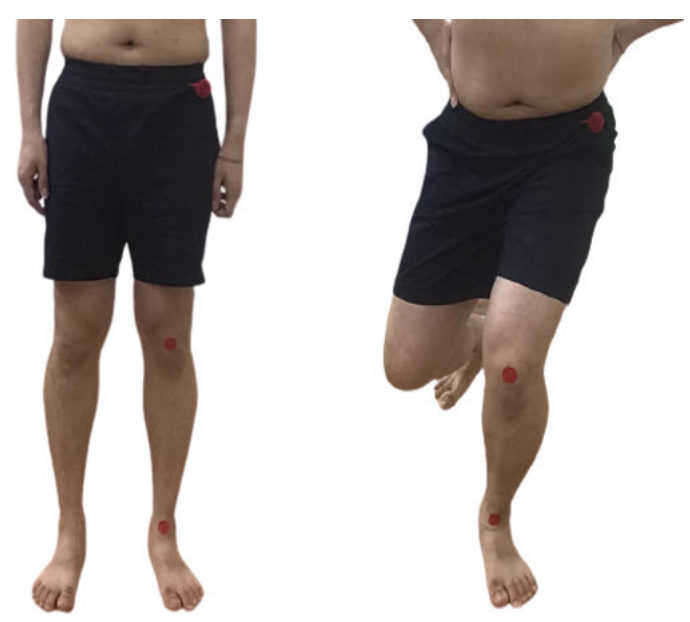

Figure 3. Single leg squat. ject was instructed to rub and press the tape attachment area for 1 minute for fixation.

\section{Muscle activity measurement}

The Noraxon Myosystem 1200 EMG (Noraxon, Inc., Scottsdale, AZ, USA), which is an 8-channel superficial electromyography, was used to measure the muscle activity during SLS (Figure 3). The sampling rate, band-pass filter, input impedance, and the common-mode rejection ratio were $1,000 \mathrm{~Hz}, 20-450 \mathrm{~Hz}, 2 \mathrm{~m}$, and $92 \mathrm{~dB}$ at $60 \mathrm{~Hz}$, respectively. The root-mean-square value was calculated using an interval of 50 milliseconds. All EMG data were processed using myoRESEARCH ${ }^{\mathrm{R}} 2.02$ software (Noraxon Inc.). The electrodes on the VMO were placed at a point 2 $\mathrm{cm}$ in the diagonal direction of the upper medial $60^{\circ}$ from the patella. The VL electrode was placed at a diagonal angle adjacent to the midline at approximately 3 to $5 \mathrm{~cm}$ above the patella. SLS was performed for 5 seconds, and the EMG values measured for 3 seconds, excluding the 1 st second and the last second, were used for data analysis. The measurements were taken 3 times, and the average was used. The values obtained from SLS were normalized as an average of 3 repeated measurements of maximal voluntary isotonic contraction (MVIC).
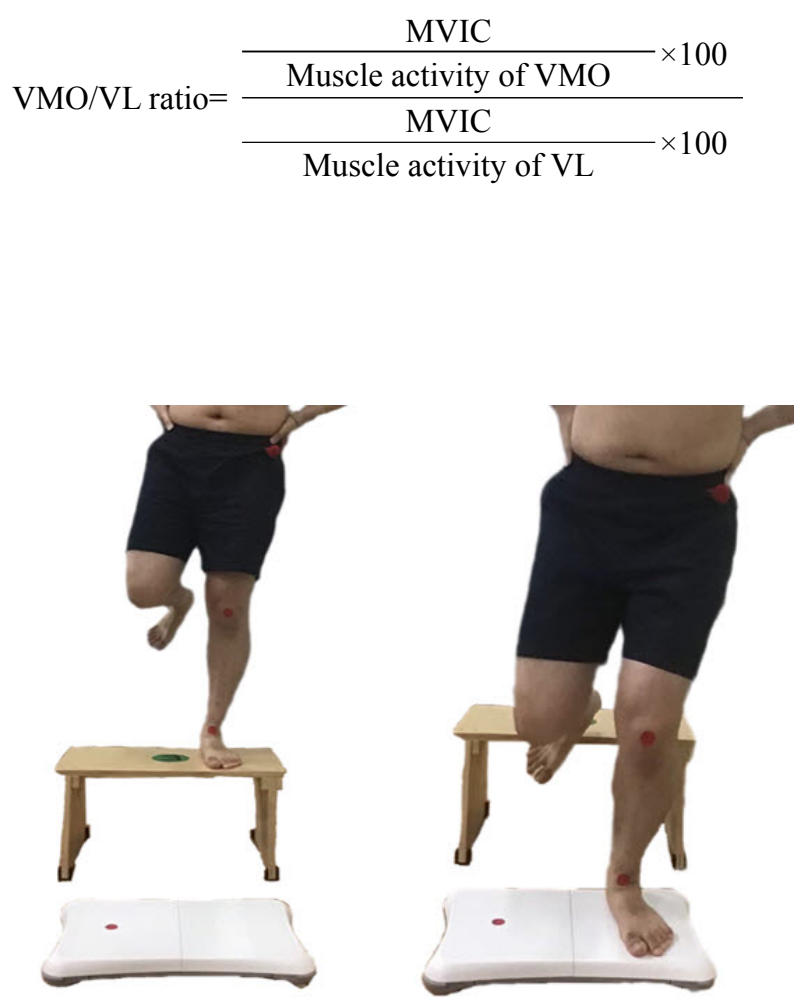

Figure 4. Single leg landing. 


\section{Vertical ground reaction force}

The Balancia program (MINTOSYS Inc., Seoul, Korea) was applied to measure the vertical ground reaction force during SLL, and a Bluetooth-connected Wii Balance Board (Nintendo, Kyoto, Japan) was used (Figure 4). The Wii balance board has a rectangular shape of $50 \mathrm{~cm} \times 50 \mathrm{~cm}$ and is used as an input device for home game consoles. The center of pressure information is collected continuously through load cells located at four corners, and information is sent to a computer device connected via Bluetooth. The highest value among the data that the subject landed on the Wii balance board on a $30 \mathrm{~cm}$-high step-box was appointed as the peak vertical ground reaction force and was normalized in proportion to the subject's weight. Three measurements were taken and the average was used for further analysis. The reliability of the Wii balance board test-retest for normal subjects was intra-class correlation coefficient $($ ICC $)=0.66-0.94$, which indicates high reliability.

\section{Knee valgus angle}

The 2-dimensional frontal projection plane angle was measured to determine the knee valgus angle during SLS and SLL. An iPad (Apple, Cupertino, CA, USA) was placed $3 \mathrm{~m}$ in front of the subject's knee level and was use to record

Table 1. General characteristics of the subjects

$(\mathrm{N}=24)$

\begin{tabular}{lc}
\hline \multicolumn{1}{c}{ General characteristic } & Subjects \\
\hline Sex (male/female) & $11 / 13$ \\
Age $(\mathrm{y})$ & $24.08(2.73)$ \\
Height $(\mathrm{cm})$ & $166.46(8.74)$ \\
Weight $(\mathrm{kg})$ & $64.00(11.39)$ \\
BMI $\left(\mathrm{kg} / \mathrm{m}^{2}\right)$ & $22.93(2.30)$ \\
\hline
\end{tabular}

Values are presented as number only or mean (SD). BMI: body mass index. through the camera application. The recorded images were analyzed using the digital video analysis software program (Dartfish 10; Dartfish, Fribourg, Switzerland). The valgus angle of the knee was measured by attaching a marker to the anterior superior iliac spine, center of the patella, and center of the ankle mortise. The knee valgus angle was analyzed by capturing the lowest point of the patella center point during the descent. The data was analyzed for an average of 3 trials.

\section{Statistical analysis}

SPSS for Windows, Version 25.0 (IBM Co., Armonk, NY, USA) was used for statistical analyses. The Shapiro-Wilk test was performed to calculate the general characteristics of the subjects and the normality of variables. A paired sample t-test was used to compare the dependent variables before and after the intervention. Statistical significance was established when $p<0.05$.

\section{Results}

Table 1 lists the general characteristics of the 24 participants in the study. No significant differences in the VMO/VL muscle activity ratio and the vertical ground reaction force during SLS and SLL, respectively, were observed after applying dynamic taping. In the case of the knee valgus angle, significant differences were observed during SLS $(p<0.05)$ and SLL $(p<0.05)$ (Table 2$)$.

\section{Discussion}

This study examined the effect of load and impact absorption of the patellofemoral joint and the effect on muscle imbalance during SLS and SLL before and after the application of dynamic taping. Significant decreases in the knee valgus angle during SLS and SLL were observed, reducing the impact and load applied to the patellofemoral joint. These re-

Table 2. Comparison of dependent variables according to the intervention within groups

\begin{tabular}{lccrr}
\hline \multicolumn{1}{c}{ Dependent variables } & Pre & Post & Diff & $\mathrm{t}(p)$ \\
\hline VMO/VL ratio (\%) & $85.68(40.66)$ & $96.01(42.34)$ & $-10.33(32.46)$ & $-1.559(0.133)$ \\
Vertical ground reaction force $(\%)$ & $3.35(0.61)$ & $3.21(0.58)$ & $0.14(0.49)$ & $1.429(0.166)$ \\
Valgus angle in SLS $\left(^{\circ}\right)$ & $20.44(10.33)$ & $16.74(9.27)$ & $3.70(6.66)$ & $2.723(0.012)$ \\
Valgus angle in SLL $\left(^{\circ}\right)$ & $19.70(6.79)$ & $16.78(5.50)$ & $2.93(6.37)$ & $2.254(0.034)$ \\
\hline
\end{tabular}

Values are presented as mean (SD).

VMO/VL ratio: VMO of \%MVIC/VL of \%MVIC, VMO: vastus medialis obliques, VL: vastus lateralis, \%MVIC: maximum voluntary isometric contraction, SLS: single leg squat, SLL: single leg landing, Diff: difference. 
sults suggested that the load and impact on the patellofemoral joint during sports activities of participants suffering from patellofemoral pain can be alleviated by dynamic taping.

Increasing the muscle activity of the quadriceps muscle is important for weight bearing, stability, and normal functioning of the patellofemoral joint [12,13]. Lee and Cho [14] examined 16 subjects with patellofemoral pain during squat exercise after applying rigid taping to fix the patella by medial gliding. As a result, the muscle activity and the muscle activity ratio of the VMO were increased significantly compared to the VL. Therefore, movement of the patella could affect the muscle activity of the quadriceps muscle. In the present study, however, there were no significant differences in the VMO/VL muscle activity ratio during SLS after applying dynamic taping. Previous studies applied a direct restriction to the patella using rigid tape, which influenced the muscle activity of the quadriceps muscle. In the present study, however, it is believed that the use of dynamic tape with elasticity did not affect muscle activity. In addition, although this study was a cross-sectional study, an effect size ( $d=0.24,95 \%$ confidence interval $=-0.33-0.80)$ was found. Hence, the application of exercise intervention, along with dynamic taping, will have more beneficial effects.

Because the peak vertical ground reaction force generated during SLL has a large impact on the whole body, a method to reduce the vertical ground reaction force is needed to perform a stable landing motion [11]. Jafarnezhadgero et al. [15] reported that after applying Kinesio taping to 20 male athletes, the vertical ground reaction force at landing decreased by $44 \%(p<0.05)$. Therefore, in this study, the peak vertical ground reaction force was confirmed when SLL in subjects with patellofemoral pain to which dynamic taping had been applied, but there was no significant difference.

This result is considered to be different from previous studies because of the taping application technique; taping was applied to the tibialis anterior muscle in the previous study to control the immediate ankle eversion, whereas it was applied only to the patellofemoral joint in the present study.

Dynamic taping applied to patients with patellofemoral pain syndrome has proven its effectiveness through several studies on the biomechanical and neurophysiological mechanisms of the patellofemoral joint during a squat [16]. Bittencourt et al. [17] reported that, as a result of applying dynamic taping to 18 female volleyball players, there was a significant decrease in the knee valgus angle from $10.5^{\circ}$ to $5.4^{\circ}$ during SLS $(p<0.05)$. In this study, after applying dynamic taping, the knee valgus angle decreased significantly from $20.44^{\circ}$ to $16.74^{\circ}$ in SLS and $19.7^{\circ}$ to $16.78^{\circ}$ in SLL $(p<0.05)$. Injury can be prevented by inhibiting knee valgus through the application of dynamic taping during sports activities that applies a loading force onto the patellofemoral joint. In several studies, it was confirmed that the application of taping during physical activities, such as walking, descending stairs, squat, and jump landing, to patients with patellofemoral pain has a positive effect because the knee motion is immediately controlled [18-21]. Accordingly, these results proved that dynamic taping performed on participants with patellofemoral pain affects the knee valgus angle during SLS and SLL.

This study had some limitations. First, it is difficult to generalize to all age groups because the study participants were limited to adults in their 20s. Second, the effects of lowering the pelvis on the contralateral side could not be controlled due to the lack of strength of the hip abductor muscle. Third, dynamic taping was applied directly to the patella so that the patella movement was not measured during the intervention. Therefore, future studies will be needed to supplement these limitations.

Dynamic taping was applied to subjects with patellofemoral pain to investigate the changes in muscle activity ratio, vertical ground reaction force, and knee valgus angle in SLS and SLL. The knee valgus angle was reduced significantly during SLS and SLL after applying dynamic taping. Overall, the application of dynamic taping during various physical activities has a positive effect on the knee valgus angle and helps reduce impact.

\section{Conflict of Interest}

The authors declared no potential conflicts of interest with respect to the authorship and/or publication of this article.

\section{References}

1. Tang SF, Chen CK, Hsu R, Chou SW, Hong WH, Lew HL. Vastus medialis obliquus and vastus lateralis activity in open and closed kinetic chain exercises in patients with patellofemoral pain syndrome: an electromyographic study. Arch Phys Med Rehabil 2001;82:1441-5.

2. Nijs J, Van Geel C, Van der auwera C, Van de Velde B. Diagnostic value of five clinical tests in patellofemoral pain syndrome. Man Ther 2006;11:69-77. 
3. Myer GD, Ford KR, Barber Foss KD, Goodman A, Ceasar A, Rauh MJ, et al. The incidence and potential pathomechanics of patellofemoral pain in female athletes. Clin Biomech (Bristol, Avon) 2010;25:700-7.

4. Elias JJ, Kilambi S, Cosgarea AJ. Computational assessment of the influence of vastus medialis obliquus function on patellofemoral pressures: model evaluation. J Biomech 2010;43:612-7.

5. Powers CM. Patellar kinematics, part I: the influence of vastus muscle activity in subjects with and without patellofemoral pain. Phys Ther 2000;80:956-64.

6. Bolgla L, Malone T. Exercise prescription and patellofemoral pain: evidence for rehabilitation. J Sport Rehabil 2005;14:72-88.

7. Bolgla LA, Malone TR, Umberger BR, Uhl TL. Hip strength and hip and knee kinematics during stair descent in females with and without patellofemoral pain syndrome. J Orthop Sports Phys Ther 2008;38:12-8.

8. Lian Ø, Refsnes PE, Engebretsen L, Bahr R. Performance characteristics of volleyball players with patellar tendinopathy. Am J Sports Med 2003;31:408-13.

9. Mostamand J, Bader DL, Hudson Z. The effect of patellar taping on EMG activity of vasti muscles during squatting in individuals with patellofemoral pain syndrome. J Sports Sci 2011;29:197205.

10. Shaheen AF, Bull AM, Alexander CM. Rigid and Elastic taping changes scapular kinematics and pain in subjects with shoulder impingement syndrome; an experimental study. J Electromyogr Kinesiol 2015;25:84-92.

11. McNeill W, Pedersen C. Dynamic tape. Is it all about controlling load? J Bodyw Mov Ther 2016;20:179-88.

12. Crossley KM, Zhang WJ, Schache AG, Bryant A, Cowan SM. Performance on the single-leg squat task indicates hip abductor muscle function. Am J Sports Med 2011;39:866-73.
13. Kim DH, Kim KH. Immediate effects of kinesio taping on muscle fatigue of quadriceps on muscle strength and balance ability. J Korean Phys Ther Sci 2020;27:43-50.

14. Lee SE, Cho SH. The effect of McConnell taping on vastus medialis and lateralis activity during squatting in adults with patellofemoral pain syndrome. J Exerc Rehabil 2013;9:326-30.

15. Jafarnezhadgero AA, Shahverdi M, Madadi Shad M. The effectiveness of a novel Kinesio Taping technique on the ground reaction force components during bilateral drop landing in athletes with concurrent pronated foot and patella-femoral pain syndrome. J Adv Sport Technol 2017;1:22-9.

16. Blyth K. Kinematic outcomes following patella taping in individuals with patellofemoral pain - a crossover design [Master thesis]. Sydney: The University of New South Wales; 2019.

17. Bittencourt N, Leite M, Zuin A, Pereira M, Gonçalves G, Signoretti S. Dynamic taping and high frontal plane knee projection angle in female volleyball athletes. Br J Sports Med 2017; 51:297-8.

18. Boling MC, Padua DA, Marshall SW, Guskiewicz K, Pyne S, Beutler A. A prospective investigation of biomechanical risk factors for patellofemoral pain syndrome: the Joint Undertaking to Monitor and Prevent ACL Injury (JUMP-ACL) cohort. Am J Sports Med 2009;37:2108-16.

19. Choi JH, Lee JK, Ahn HJ. The effect of a kinesio taping on muscle power and muscular fatigue in quadriceps femoris. J Korean Phys Ther Sci 2008;15:19-26.

20. Jung BO. The effect of kinesio taping on the quadriceps femoris to the gait characteristics. J Korean Phys Ther Sci 2008; 15:6572.

21. Yang KH, Han JM. Kim SH. The effect by taping therapy for pain of knee joint. J Korean Acad Phys Ther Sci 2006;13:35-41. 